\title{
Einstein: Unification Theory and Unification of Basic Forces-II
}

\author{
M. Tajuddin ${ }^{1}$, Md. Haider Ali Biswas ${ }^{2}$ * \\ ${ }^{1}$ Directorate of Primary Education, Dhaka, Bangladesh \\ ${ }^{2}$ Mathematics Discipline, Khulna University, Khulna, Bangladesh
}

Email address:

dmtajuddin@yahoo.com (M. Tajuddin), mhabiswas@yahoo.com (M. H. A. Biswas)

\section{To cite this article:}

M. Tajuddin, Md. Haider Ali Biswas. Einstein: Unification Theory and Unification of Basic Forces-II. American Journal of Physics and Applications. Vol. 3, No. 5, 2015, pp. 166-177. doi: 10.11648/j.ajpa.20150305.12

\begin{abstract}
Unification theory is 'a complete unified theory that will describe everything in the universe' (Hawking, 1996). Unification theory is expressed through spherical geometry and 'all dimensional geometry is included in spherical geometry. Thus spherical geometry in the form of unification theory describes all the laws of the universe' (Tajuddin and Biswas, 2015). This theory has enough proofs and supports in favor of its formation and working capacity. The unification theory and spherical geometry are not any type of utopian ideas but they are basic and real concepts of the world that are searched by our scientists for the last hundred of years. Actually this unification theory is the Grand Unified Theory (GUT) of four basic forces of physical sciences, and spherical geometry is the supreme geometry that covers the unified force. These two concepts are related to each other and discussed here (vide: Different sections of the article).
\end{abstract}

Keywords: Theory of Unification, Unified Force, Spherical Motion, Spherical Geometry

\section{Introduction}

What is long range force? What is short range force? Long range particles come from the distance and short rage particles remain in the inertia of mass. The union of two groups creates unified force. What is unified force? 'Every particle feels the force of gravity according to its mass or energy' (Hawking, 1996). 'So the long range forces have appeal to the short range forces in inertia of mass of the earth. The long range virtual particles discharging from the sun induce to three basic forces of inertia of mass of the earth and the short range forces sustain that force and thus results in unified force'-(Tajuddin and Biswas, 2015). Here gravitation and its allied lepton group virtual particles discharging from the sun are known as long range particles that interact with the short range forces belong to hadrons group lying in the inertia of mass of the earth and other planets, and create unified force that controls the universe and other activities of the world in spherical motion. This bondage is the high powered interaction in the universe in between long range particles of spin $1 / 2$, and short range particles of spin 1 and 2 of electromagnetic and weak and strong nucleus forces respectively. The bondage of the two groups creates unified force and unless there is bondage, gravitation may not interact with the opposite hadrons group and the universe will not work (vide: section-3). The function of unified force is separate from the functions of each of three basic forces such as electricity shows how two opposite charges meet together and how current flows, and electromagnet shows electromagnetic induction and wave in the space, and weak and strong nuclear forces 'binds particles together into combinations' (Hawking, 1996) but unified force shows interaction of long range forces with short range forces, and how long range force absorbs in nucleus of atom and gives reproduction in the form of new electron in atom and 'make up the matter in the universe' (Hawking, 1996) and creates unified force and controls the universe (vide: sections-2,3,4). Thus physics has close affinity with biological sciences by unification theory as regards breeding though it happens differently. Another aspect is that long range particles may be used as more active agent other than electron of electricity because we consider that long range particles are the most tiny particles.

Unified force has microcosmic and macrocosmic representation of the world. Earth moves around the sun, universe has itself spherical motion, which is microcosmic representation but electron as well as wheel of vehicles has spherical motion which is macrocosmic representation of unified force. Unified force joins two far-fetched forces 
together and both the forces behave in the same way. Here lies the credibility of unified force.

We shall find, unification theory has two parts such as first, gravitation interacts with three basic forces and summation of three interactions create unified force (vide: section-16) and second, unified force creates spherical motion that grows stronger and controls the universe (vide: section-17, 21.6). Summation of interactions of three basic forces will go to the credit of unified force. The long range forces move spherically and interact with short range forces and create unified force that controls the universe and all motions of the world. Here metrics of unified force have been developed on spherical motion under spherical geometry 'that will describe everything in the universe' (Hawking, 1996).

\section{Unified Force}

'Unification theory is expressed here by quantum mechanics and theoretical physics interdependently in the elementary level. 'Einstein wished to relate all the properties of matter in a unified field theory' (Strathern, 1997). Einstein found that space-time is curved and gravitation is also curved and they curve all motions. But he could not succeed in his attempts. According to Einstein's equation $\mathrm{E}=\mathrm{mc} 2$ inertial mass of the earth equals to total energy of the earth. In theoretical physics, properties of matter include four basic forces of quantum mechanics. The earth is a big inertial mass with spherical atmosphere. The earth with all properties of matter and basic forces moves around the sun. Again in quantum mechanics 'Einstein's main scientific interest was in developing a unified theory, an attempt to explain both gravity and electromagnetism in one mathematical package' (White and Gribbin, 2005).In 1920s, the only two forces known to physics were gravity and electromagnetism, but very little was known about the nuclear forces. In the circumstances he developed law of gravitation where a small particle moves around the sun in empty space. But Einstein could not succeed in both the attempts because we know from his system of approach to metrics and Schwarzschild solutions that he used Euclidean type of zero tensors in his metric.

The sun has rotational and orbital movements but due to the rotational movement the long range virtual particles discharging from the sun reach the earth spherically to three basic short range forces such as electromagnetic, weak and strong nuclear forces lying in the inertia of masses of the earth. 'Every particle feels the force of gravity according to its mass or energy' (Hawking, 1996). So the long range forces have appeal to the short range forces in inertia of masses of the earth. The long range virtual particles discharging from the sun induce to the three basic forces of inertia of masses of the earth and the short range forces sustain that force and thus results in unified force.

The long range particles spherically interact with the short range three basic forces lying in the inertia of mass of the earth and create unified force. When the interaction of gravitation works, simultaneously the unified force also starts functioning to control the earth with its vast inertial masses around the sun. This unified force controls the universe and all activities of the world in spherical motion. The unified force is formed by four basic forces of quantum mechanics as well as by theoretical physics and in both the cases some extra properties such as radius of circle, cyclic frequency, mass of the body and time join unified force to make it workable. (Vide: Structure of unified force).

Now the question arises, why and how the gravitation as long range force interacts with three basic forces lying in atom? Gravitation interacts with three basic forces to make them fertile because atom has to develop itself adding more electrons in its body and gradually take different shapes suitable to 102 elements. Thus the smallest atom of hydrogen having one electron and one proton and nucleus develops into different heavy atoms of different elements. Here gravitation interacts with basic forces of atom as that of pollination in botanical sciences. Inanimate substance increases absorbing heat and water day by day. 'The laws of nature remain covariant independent of the frame of reference' (Prakash, 1985 ). We use synonym words in different discipline such as interaction in physics, pollination in botany, sex relation in zoology etc. Sometime it happens for keeping the universe active and viable, otherwise planets would not move, the universe will not work. The solar system will not work unless the sun gives incentive to the planets for their motivation; the case is the same every where. The husband gives wife motivation in the form of food, nourishment and children, and the wife works in return and response. When two charges meet together, current of electricity flows but in case of unified theory when the long range particles interact with the short range particles, nucleus fertile and new electron comes of in the lap of mother atom and new element creates. Thus the flow of electricity in between two charges, and interaction between long range particles and short range particles are different. What is the difference between electricity and interaction of unified force? Electricity gives us flow of current but produces nothing, on the other hand interaction of unified force produces new babe in the form of new electron in atom and contributes world process of development. Physics is not as a dull subject as we think. The case is the same in biological sciences, physics, chemistry, economics, political sciences and others. The motto is that nothing comes out of nothing. Thus unified force proves its own existence, functions and geometry.

\section{Unification of Elementary Particles}

As we find there remains gap of communication in between the two groups of particles such as lepton group (electron, moun, neutrinos, tau etc,) particles and hadrons group (nucleons, pions etc,) particles. It remains as a hurdle for building unification theory. Unification theory overcomes the hurdle. Quantum mechanics discovered 'All the known particles in the universe can be divided into two groups: 
particles of spin $1 / 2$, which makes up matter in the universe, and particles of spin 0,1 and 2 which, we shall see, give rise to forces between the matter particles' (Hawking, 1996).How relation is made between the two groups? The first category of lepton particles which have no apparent internal structure that makes up matter in the universe but how they find materials? The materials lie with the second category of hadrons particles which is massive, short range and stays inside the atom. Thus the interaction is necessary between the two groups. The first category of particles like lepton group and quark group having spin $1 / 2$ and spin $1 / 3$ respectively are long range particles spherically come across a far distance and interacts with second category of particles lying in the inertia of mass of the planets and create unified forces that controls the universe. This is the highest interaction in the universe. Thus unification theory coordinates between long range particles and short range particles and unifies them under the banner of unified force.

\section{Gravitation and Three Basic Forces}

Maxwell used the four basic laws of electricity and magnetism and predicted that energy of electric and magnetic fields can be propagated in the form of waves, called electromagnetic waves, the velocity of which was calculated and found to be equal to the velocity of light, (Vasudeva, 1983). Unified theory expresses that gravitation interacts with three other basic forces of inertia of mass. So the gravitation interacts with the electromagnetic force, weak and strong atomic forces lying in the spatial atmosphere of the earth. It is impossible to spread electromagnetic wave all over the world without influence of gravitation. There should be two opposite charges that attract each other. The long range particle disperses from the sun due to atomic fusion in the sun and after crossing a long way induces to three basic forces of inertia of mass of the earth and helps the earth to move around the sun. According to Maxwell's equation, electricity and magnetic force induces to each other and propagates electromagnetic waves all over the world. On the other hand, according to unified force the long range particles dispersed from the sun interacts with three basic forces in the inertia of mass of the earth and revolves the earth around the sun. As we know the electromagnetic force is short range, so it cannot propagate electromagnetic wave all over the world. Ohm, Coulomb and Gauss show how two opposite charges meet together and current flows, and Faraday shows induction of electromagnet and Maxwell shows electromagnetic wave in the space and weak and strong nuclear forces show how it binds particles together into combinations, but unified force shows interaction of long range forces with short range force, and how long range force absorbs in nucleus of atom and gives reproduction in the form of electron and contribute making up the matter in the universe (vide: section-3). Here the function of unified force is shown separate from two electric charges, electromagnetic induction, electromagnetic wave and nuclear's combination of particles. Gravitation performs three types of works with three basic forces. First, it interacts with three basic forces lying in inertia of mass of the earth and formed unified force that helps reproduction of matter and functioning of the world process. Second, it interacts with electromagnetic force and propagates electromagnetic wave in the space. Third, it works with weak and strong nuclear forces to bind the structure of the particles confirmed in atom. 'The strong nuclear force has a curious property called confinement: it always binds particles together into combinations' (Hawking, 1996). The constituent formula of three basic forces are different from each other but the interaction of gravitation with three basic forces happen uniformly, spherically, simultaneously and works in specific direction, and here lies the unification theory and unified force.

\section{Gravity and Gravitation}

The long range virtual particles derived from the sun are induced spherically to the enclosures of short range particles of three basic forces lying in atom of inertia of mass of the earth and tightly catch with them. The string of gravitation is long but the catchment with short range forces is tight. The catchment is as tight as the ships anchor in the vast ocean in violent storm. Gravitation has interaction with each of the three basic forces and creates bondage with them in the form of unified force and as a result unified force is stronger than any of the basic force. Thus gravity is created when the long range particles are induced spherically to three phases of short range forces in the nucleus of atom in the earth. The long range particle in the process of attraction with short range particle is known as solar gravity. The earth has the same type of particles known as earth gravity that revolves the moon around the earth. The mutual interaction between the sun and the earth is known as gravitation. Now let us know how unified force is created? The long range particles spherically interact with the short range three basic forces lying in the inertia of mass of the earth and thus results in unified force.

\section{Trend of Physics}

Galileo was the pioneer of modern physics but 'proper idea of gravitation was absent from science at the time of Galileo. Therefore, the influence of gravitation was not incorporated with the laws of falling bodies, simple pendulum etc. of Galileo. This affects adversely physics afterward. Galileo mentioned in the first law of falling bodies that in vacuum all bodies, starting from rest fall with equal rapidity. Here the phrase 'equal rapidity' affects adversely physics and mathematics. Why do two bodies; one big and one small, fall with equal rapidity? In physics, it has no proper answer. Does gravitation attract each body equally irrespective of mass? Does gravitation attract each body proportionate to the mass of the body? Firstly, if gravitation attracts equally each body, masses of all bodies would be equal, but the masses of all bodies are not equal. Secondly, if 
gravitation attracts all bodies proportional to mass of the body, all bodies would fall with equal rapidity and simultaneously and their masses would differ from each other. This idea is absolutely right. Galileo drew the idea of equal rapidity from falling bodies comparing with one another without considering the influence of gravitation. Attraction of gravitation was not incorporated with falling bodies. Actually gravitation influences upon all bodies proportionate to the mass of the bodies and as a result all bodies fall with equal rapidity. Here it is better to say 'proportional rapidity' instead of 'equal rapidity' because gravitational attraction is proportional to the mass of the body. As gravitation was not incorporated with the laws of falling bodies and as a result afterward gravitation is omitted from all laws of motion. In the same way Newton followed Galileo in his 'Experiment of Guinea and Feather' as body falls with equal rapidity. Newton draws his law of inertial motion without the influence of gravitation and as a result laws of inertia have become linear. He stated in his first of motion that 'Every body continues in its state of rest or of uniform motion in a straight line unless compelled by some external force to act otherwise, (Abbott, 1997). His law of inertia and law of gravitation are linear. Newton aggravated the situation developing laws of inertia and law of gravitation separately. But now-a-days it is clear that gravitation influences upon all inertial masses. There would be a common law joining inertial law and gravitational law together that would be unified law.

In twentieth century Ronald Von Eotvos of Budapest, Robert Dicke of Princeton University and Igor Braginsky of Moscow made an attempt to discover the ratio of inertial mass and gravitational mass but they could not take correct decision because the elements of their experiments were somewhere gold and aluminium and somewhere platinum and aluminium cylinders. These elements were not in consonance with that of Galileo in Leaning Tower of Pisa. There was a criticism about those experiments that 'the mass tested were small and the possibility remained that heavy objects might behave differently' (Calder, 1979). Thus the explanation of equality of mass was not given correctly. The answer is that body gets mass from gravitation proportional to the mass of the body and this is why the gravitational mass and inertial mass are proportional. But it is not properly explained in physics.

Einstein was the pioneer of unification theory but he followed the path of his predecessors in his principle of equivalence that 'different masses fall at the same rate' (Good, 1974). Einstein 'exhibited a remarkable relationship between gravitation and inertia what is expressed in the law of the equality of gravitational and inertial mass' (Born, 1962). In principle of equivalence gravitation and inertia mass are considered as equal. If gravitation and inertia mass are considered equal how gravitation would control the universe? Thus Einstein's principle of equivalence is a hindrance to unification theory and it also goes against the spirit of spherical geometry. Einstein framed law of gravitation where a small particle moves around the sun in empty space. Einstein followed zero tensor in his metric and developed zero tensor in law of gravitation in empty space. 'This is empty space without electromagnetic field and without matter' (Hawking, 2007). In fact, world of empty space was fictitious. There exists no such world. Greeks first developed linear equation and afterward Newton arranged equation of empty space in his third law of motion $(\mathrm{F}=0)$. Einstein followed him in his zero tensor and developed law of gravitation using zero tensor and commits wrongness. 'The resultant force on a body can never be zero and it is always in the form of acceleration' ( Tajuddin and Biswas, 2005). Einstein had an attempt to nonlinear metrics but he failed. Thus he could not overcome the classical mechanics though he had consistent thoughts about the universe. Einstein sometime bents towards classical mechanics and sometime towards spherical geometry and lost his way in search of unification theory (Tajuddin and Biswas, 2015).But we are to reach the highest level of unified force and unification theory.

\section{Trend of Mathematics}

The ancient 'Greek had a belief that the earth was stationary and that the sun, the moon, the planets and the stars moved in circular orbit about the earth' (Hawking, 1996). Greek mathematician Euclid devised Elements considering the earth as flat and static and his geometry subsequently developed by Descartes is appropriate to two and three dimensional world. In seventeenth century the concept of earth is changed as it is found spherical and has rotational and orbital movement. 'Thus Euclidean Geometry was no longer sufficient to describe the universe' (Strathern, 1997). Einstein asserts 'the geometry of the real world is actually not Euclidean' (Born, 1962). Since Euclidean geometry failed, Einstein could have fallen back on some other definite non-Euclidean geometry. Gauss had sketched out the theory of curved surfaces in the form of a general two-dimensional geometry and Riemann had extended this doctrine to continuous manifold of any number of dimensions. The theory was further developed by Christofell, Ricci and levi-Civita, among others' (Born, 1962). There are only two ways of contracting Riemann-Christofell tensor, one way leads to Ricci tensor and the other way leads to zero tensor. Ricci tensor further was contracted with Bianchi Identities. But at the pole of geodesic coordinates system, both kinds of Christofell's symbols vanish but not necessarily their derivatives also, (Prakash, 1985). Einstein works with Bianchi Identities which turns to zero tensor and commits the same fallacy of his predecessors. We know also from Schwarzschild solutions that Einstein worked with zero tensor. This is why 'the general theory of relativity is called a classical theory' (Hawking, 1993). Zero tensor is traditional equation where in the case of motion, action and reaction are equal and opposite (Example: $\mathrm{F}=-\mathrm{F} / \mathrm{or}, 2 \mathrm{FF}=0$ ). Thus Euclidean geometry or Einstein's metric are not the proper geometry that can solve the unification theory. But we are to establish the spherical geometry with unified force for the 
solution of the problems of physical sciences..

Riemann developed N-dimensional coordinate from three dimensions. But it's a matter of regret that the prospect of Riemann was not understood by mathematicians of his age or even today. Contemporary mathematician comments about Riemann that 'Riemannian curvature is equal to the Gaussian curvature' (Synge and Schild, 1969), which was not right because Gaussian tensor is curvature tensor of surface and Riemannian tensor is round coordinates of space. Further converting Riemannian coordinates to RiemannChristofell tensor was not a right direction. N-dimensions of geometry have immense possibility for spherical geometry. We shall find in our study that when fixed numbers are added with each Cartesian coordinate $(\mathrm{x}, \mathrm{y}, \mathrm{z})$, it becomes $\mathrm{N}$ dimensional coordinates. Adding time with $\mathrm{N}$-dimensions, it gets spherical motion. This spherical motion of spherical geometry may solve unification theory. This spherical motion stands to single dimension. This metric is known as line element which is used in spherical geometry.

\section{How Body Gets Mass}

Attraction of gravitation is an important factor by which body gets mass and spherical motion. When body gets mass, it has spherical motion. The planets have spherical motion because they have mass in the form of gravitational attraction. According to the theory of relativity, 'Gravitational and inertial mass are equal' (Born, 1962). Weight of the body is the combined attraction of gravitation and gravity. 'Here gravitational mass simply signifies the weight divided by ' $\mathrm{g}$ ' and the proper mass is distinguished by prefixing the word 'inertial'-(Born, 1962). Body gets mass through interaction of gravitation with three basic forces of inertia and hence the two masses are called equal. This proves that body has no mass of its own, it gets mass from gravitation. Thus classical mechanics considers gravitational mass and inertial mass are equal. But according to unification theory body gets inertial mass from gravitation proportionate to the mass of its body and thus it is named as proportional mass instead of equal.

\section{Disorder in the Laws of Motion}

Laws of physics do not follow any ultimate principle and as a result the laws sometimes do not perpetuate and everlasting. Theory of Aristotle and Ptolemy about solar system has been exhausted. There still exists disorder in the system of laws of motion and gravitation as one system of laws does not adjust with other system. The classical laws of motion are linear as it is stated in the first law 'Every body continues in its state of rest or of uniform motion in a straight line unless compelled by some external force to act otherwise' and in third law 'to every action there is an equal and opposite reaction' (Abbott, 1997). But the law of gravitation is spherical as expressed by Einstein where a small particle moves around the sun in empty space. Maxwell's equation predicted electromagnetic wave that spread light all over the solar system but electromagnetic force is a short range force and how it can disperse light all over the solar system? On the other hand the sun is considered as a big electromagnet that disperses light itself, and gravitation interacting with electromagnetic force creates wave all over the solar system. Sometime the idea of electromagnetic wave and gravitational interaction are coincided with each other but the idea of unified force should be upheld. The spherical motion and spherical geometry may bring adjustment in the laws of motion and gravitation.

\section{Unified Force Works to Spherical Motion}

Unified motion works in spherical motion. Spherical motion is created in two ways: one is application of force and other is attraction of gravitation. Motion becomes spherical when it passes from high pressure to low pressure, dense medium to light medium and vice-versa. In both the cases advantage is the same. Motion also becomes spherical if it passes from a vacuum to air, from slow speed to high speed or from motion to rest and vice-versa (Born, 1962). The engines of vehicles, factories and aircraft create motion with high-pressure to low-pressure techniques. Wind, cyclone etc. blow from high pressure to low pressure. Spherical motion is found in the laws of magnet, electromagnet, induction, magnetic field, and gravitation, application of force and refraction of light. Gravitational attraction is spherical as the earth moves around the sun. It is noted here that single change of motion from dense medium to light medium causes curvature in a point, that is, refraction of light but frequent changes cause spherical motion as wheel of vehicles. When vapour of engine of vehicles such as aeroplane, bus, train etc., passes from high pressure to low pressure frequently in a same point, wheel or body moves spherically pushing (friction) with air, road or rail slipper respectively and the vehicle moves swiftly (Fig-1). When light passes from light medium to dense medium, it curves and creates motion (Fig-2). Motion from dense medium to light medium creates swift motion. Here air is light medium and glass is dense medium. Motion passing from light medium to dense medium and high pressure to low pressure possesses same advantage (vide: Principle of spherical motion). Nature expresses itself in different ways. Aeroplane gets motion in the runway as its engine works in high pressure to low pressure and flies in the sky with swift motion overlooking the gravitation. Here overlooking the gravitation means overlooking the mass of the body also because mass derives from gravitation. Plane flies straight due to joining wings but its engine works spherical. Buses run straight on the road but engine works spherical and so on. When rifle is fired, it creates swift motion in the same way. If a motion curves every moment, it becomes a spherical motion (vide: spherical geometry). Pressure in engine from high pressure to low pressure creates spherical motion with acceleration. There is no difference of motion in 
principle between flying of plane, moving of busses and refraction of light. Example of spherical motion is shown in high pressure to low pressure in engine of aeroplane and bus or dense medium to tight medium in refraction of light in figures- $1 \& 2$ respectively and riding and walking by a boy and a girl in figure-3.

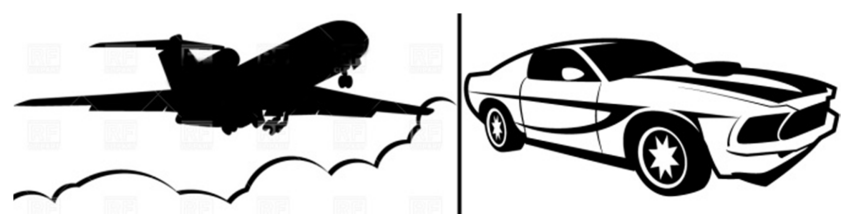

Figure 1. An aeroplane flying and a car moving.

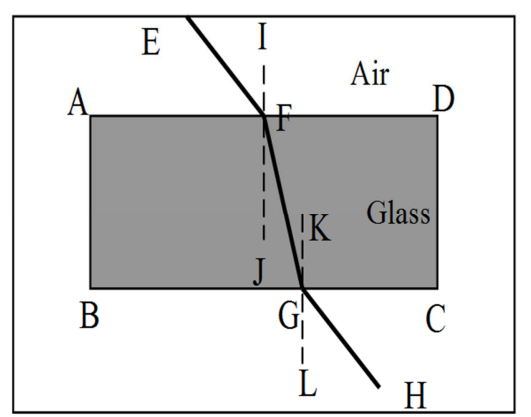

Figure 2. Refraction of light.
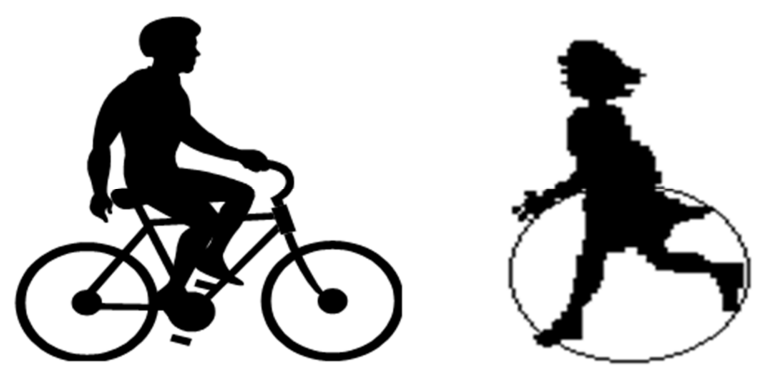

Figure 3. A boy rides bicycle and a girl walks.

In figure- 3 the boy rides bicycle once pressing pedal by right foot and then pressing pedal by left foot in sequence of spherical motion. On the other hand the girl walks lifting her right leg and then left leg in sequence of spherical motion. Is there any difference between riding and walking? Answer is no. Walking and running of animals and flying of birds respectively can be explained in the same way in spherical motion. Now it is clear with these proofs and supports that motion is always spherical in nature, never linear in spirit. We want to say again that nature expresses same phenomenon of motion in different ways.

\section{Principles of Spherical Motions}

In the case of application of force or gravitation, the motion or attraction respectively, passes from a dense medium to a light medium that would be considered high pressure to low pressure ( $1^{\text {st }}$ case). If the motion or attraction passes from a light medium to a dense medium that would be considered as low pressure to high pressure $\left(2^{\text {nd }}\right.$ case $)$. In the first case, due to the application of force, the spherical motion would be counter-clockwise and due to attraction, the spherical motion would be clockwise. The motion in both instances would be forceful and accelerated. In the second case, due to the application of force, the spherical motion would be clockwise and due to attraction, the spherical motion would be counter-clockwise. The motion in both the instances would be mild and accelerated. In nature the left movement and the right movement of motions are visible but the left movement is prevalent.

\section{Mechanical Advantages}

Physics has a chapter known as mechanical advantage and mechanical advantage has no link with law of motion. Works of daily life are done by the professionals with the help of mechanical advantages. Professionals like farmer, black smith, potter, barber, fisherman, washer man etc. perform their duties with the help of mechanical advantages. Vehicles like bus, train, plane etc. move or fly with the support of an engine where mechanical advantages work. Crane is used in seaport for shipment of goods. Mechanical advantage is nothing but using of spherical motion of unified force. Unified force works here as mechanical advantages. The smaller cases may be called mechanical advantages but the bigger cases are unified forces. The sun has gravity but it is not sufficient to handle the huge planets like the earth and the Jupiter without the help of unified force. Thus the universe with all activities is controlled by the unified force. Einstein desires such type of force.

\section{Uniformity of Motion}

Nature has diversity but it has uniformity in one point of spherical motion. Again spherical motion expresses itself in different ways. As we know all motions and activities of the universe happen in spherical way. So nature is uniform in respect of all spherical motions of the universe. Nature performs its works with uniformity and discipline. Every day sun rises in the morning and sets in the evening, which stars are seen in the evening, decline in the last night, tide, ebbtide and flow of current in the ocean, changes of seasons, birth, growth and decay of life happen in natural courses. Natural courses happen in spherical motion and timetable, so nature is uniformed. Nature is so uniformed and disciplined that mankind prepare calendar to follow natural courses. Natural course is uniform in the point of motion is shown in figure-4.
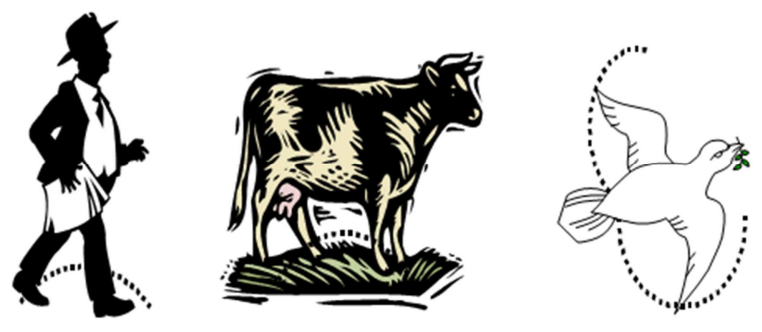

Figure 4. A man walking, a cow running and a bird flying. 
Here a man is walking, a cow is running and a bird is flying in spherical motion. Is there any difference among these movements in respect of motion? Answer is in the negative.

\section{Reaction is Greater Than Action}

The earth is elliptical, space-time is curved and gravitation is also curved and it curves all motion. 'Gravity and spacetime is curved or warped',-(Hawking.1996). Bus, train and vehicle run on wheels. The engines of planes, ships and vehicles work with high pressure to low pressure which is spherical in motion. Therefore all motions move spherically. If the motion works spherically, reaction would be greater than action. In previous day it was not known that gravitation has influence on all inertial masses but it is now clear that gravitation influences on all bodies. In classical mechanics laws of motion is considered as: action and reaction are equal. 'The resultant force on a body would always be zero, (Resnick and Halliday, 1966 ), but this idea is changed nowa-days. Now we are to follow reaction is greater than action. 'The resultant force on a body can never be zero and it is always in the form of acceleration or retardation' (Tajuddin and Biswas, 2005)

\section{Philosophy of Spherical Motion}

The earth is elliptical and it has rotational and orbital movements. All stars, planets and satellites have spherical motion. If we minutely observed, we shall find that all activities and movements of men and animals are spherical. The motions of vehicles and machines move on wheels or motors are spherical. Ancient people discovered wheel that are very consonance to spherical motion. Every object in the universe has a tendency to lie in balance shape because gravitation exerts an influence upon them equally. If a body lies in balanced and another body lies in round shape, what is the difference in their position? They have the same advantage in both positions. All bodies in the universe- animate and inanimate take same advantage in their movement and it is spherical motion. All reactions are a like. The applied force or attraction helps the round body to move with ease and spherically. Here mechanical advantages work, applying less force getting more reaction. If force is applied to a around shape body or balanced body their techniques of movement is different but advantage is the same. Suppose, animal walks, birds fly, vehicles rotate, earth moves around the sun etc., have the same advantage of spherical motion. This is the philosophy of spherical motion. Philosophy of spherical motion of a moving body and a balance is shown in figure-5.

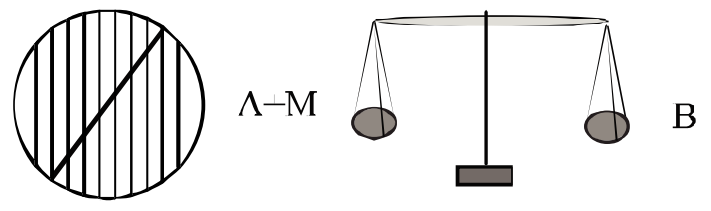

Figure 5. A moving body and a balance.

\section{Interaction of Unified Force}

How gravitation interacts with three local forces such as electromagnetic, weak and strong nuclear forces? Three forces will not work unless gravitation interacts with them. We have a lot of proofs in favor of the above argument such as hydrogen as a light atom lies on the surface of the atmosphere as the long range particles interacts with and absorbs in hydrogen atom and gradually fertilizes its body joining more electrons suitable to different elements. Plants prepare food in leaves with the help of sun rays called photosynthesis. Seeds cannot germinate without sun ray, water and air. Water absorbs heat and become vapor. Earth, rocks and matter change shapes day by day absorbing heat and water. Three basic forces lie every where in the earth such as in atmosphere, water, fauna and flora and surface of the earth, interacts with them and gradually changes shape. In case of fauna and flora food habit is important for them but it is not all. Long range particle has influence on all living beings in their birth, growth, decay and death. What so ever, quantum mechanics have analyzed the interactions of three short range forces, but this will go in consonance with interaction of gravitation. Thus the interaction of basic forces works in consonance with interaction of long range forces. As basic forces lie everywhere, so long range forces in the form of gravitation works everywhere.

As we know three basic forces harbor in the nucleus of atom in inertia of mass. 'Recently, successful attempts have been made to unify three kinds of the four categories of force'(Hawking, 1996). The fourth category is gravitation which is a virtual particle can not be detected by photon detectors. 'This force is universal, that is, every particle feels the force of gravity according to its mass or energy...However, the fact that it is both long range and always attractive means that its effects all add up. So far a sufficiently large number of matter particles, gravitational particles can dominate over other forces. This is why it is gravity that determined the evolution of the universe, (Hawking, 1996). As per above observation, gravity as a long range particle interacts with short range three kinds of particles such as electromagnetic, weak and strong nuclear forces lying in the inertia of mass that results in unified force. Summation of interactions of four basic forces may be called Grand Unified Theory (GUT) which is termed here as unified force. Combining the interaction of four basic forces we get unified force as $10^{3}$ times stronger than the electromagnetic force. Thus gravitation is the weakest and unified force is the strongest of the five forces (Goldstein, 1999). In support of spherical interaction, unification theory has been designed that controls the universe with spherical motions of all kinds which is assigned in favor of spherical geometry (vide: sections-17, 21.6).

\section{Formation of Unified Force}

As attraction or application of force always derives spherically from same sources and same system, and thus motion becomes spherical, otherwise universe would not 
work. Again unified force works spherically and grows stronger (vide: section-10, 11). Leptons group particles are virtual particles and as a result they cannot be specified by metal detectors. We need not to wait for the visual discovery of virtual particles for unified force because unified force is important for us for our advancement and we have the results of virtual particles. These virtual particles may be or may not be observable in near future. Though virtual particles are not detected but natural phenomena caused by virtual particles are visible. If we can discover the natural effects, we can know the natural causes also. There is relationship between cause and effect of natural phenomena. Sometime we can not observe causes of natural happenings but we are well aware of the effects of such causes, such as earth's spherical movement, eclipse, earth quake, cyclone, tidal bore, engine works, vehicles' movement, plane flies, air blows, water falls etc., due to spherical impact of gravitation with three basic forces. We have the results of virtual particles in our hands. Thus unification theory is developed perceiving the universe intuitively and observing the effects created by natural causes along with matrices of spherical motion with logical and logistics supports and mathematical interpretation and analysis reports. Therefore, we may conclude that discovery of unification theory is right and unified force is right, and analysis by spherical geometry is also right (vide: section$21.5,21.6)$.

\section{Necessity of Unified Force}

It is important to know the unified force because it controls the universe. 'Unified force derives from spherical motion of the universe and they develop spherical geometry. Spherical geometry includes all dimensional geometry. Thus spherical geometry in the form of unification theory describes all laws of the universe'( Tajuddin and Biswas, 2015). Thus unification theory includes all physical laws under its domain with systematic and gradual developments and, which law would not be included might be omitted as a fake law. Unification theory joins one physical law with another systemic way but existing study does not aim at this way. Thus unification theory may be used as an ultimate scale to test all scientific laws and formula and to find out undiscovered laws. Thus all scientific laws and formula will be under one line. Our scientists know more or less different happenings of the world but this theory will bring all learning in one line. This theory will help us to know the bondage of gravitation and three basic forces lying in inertia of mass, to know the techniques of unified force how to control all motions and activities of the universe, to know the cause of cyclone, tidal bore, hurricane, eclipse, earth quake and other natural happenings, to know how body gets mass and spherical motion from gravitation, to know the spherical motion from high pressure to low pressure, dense medium to light medium under gravitation and vice-versa, to know how motion passes from a vacuum to air, from slow speed to high speed or from motion to rest and vice-versa under gravitation, to know how bodies in vacuum, starting from rest, fall with equal rapidity, to know the principle of proportionate between gravitational mass and inertial mass and so on. Actually unification theory may work as a touching stone in physics and mathematics. This theory may help to conclude the scientific projects in Dallas in USA and in Jura Mountain in Switzerland and proceed further with new dimension. So it is important to know the unification theory.

\section{Up-dating Curriculum}

Our physical sciences are full of back dated theories and concepts. These theories have little linkage with modern sciences. "The rate of progress is so rapid that what one learns at school or university is always a bit out of date,' (Hawking, 1996). Our young generation studies these wrong theories from their curriculum. Without standard curriculum and studying backward theories and concepts, how can our young learners think about latest innovations of science and technology? Therefore, it is needful to up-date the curriculum of physical sciences all over the world

\section{Mathematical Analysis}

Mathematicians developed geometry from the dawn of civilization as they conceived the world. As the civilization advanced so the geometry developed. So far five kinds of geometry have been developed. These are as follows: Twodimensional, Three-dimensional, Non-Euclidean, Fourdimensional, Spherical geometry and Spherical geometry of Unified Force. Ultimately we find spherical geometry suitable to measure the spherical motion of all kinds including spherical motion of long range particles and spherical motion of the planets etc.

\section{Two-Dimensional Geometry}

About three thousand years ago when wonderful civilization was flourished in Greece, people had a belief that the earth was the centre of the universe and all the heavenly bodies moved around the earth. The earth was stationary and flat. Greek mathematicians like Euclid, Pythagoras and Archimedes developed mathematics considering the earth as stationary and flat. Euclid wrote Elements taking the scattered ideas of geometry of the age. His geometry is known as plane geometry which considers ten postulates such as dot, line, straight line, plane etc. Plane geometry has two dimensions such as length and breadth $(\mathrm{x}, \mathrm{y})$. But the concept of earth is changed in seventeenth century as it is spherical and has rotational and orbital movements. 'Thus Euclidean geometry was no longer sufficient to describe the universe' (Strathern, 1997). Here spherical geometry is necessary. Two dimensional coordinate is shown in figure-6.

$$
\begin{aligned}
& \text { Breadth } \\
& \text { Length }
\end{aligned}
$$

Figure 6. Two dimensional coordinate. 


\subsection{Three-Dimensional Geometry}

In seventeenth century Descartes developed Euclidean plane geometry into three-dimensional geometry. This plane geometry has three dimensions as length, breadth and height. Three dimensional coordinates $(\mathrm{x}, \mathrm{y}, \mathrm{z})$ is shown in figure- 7 .

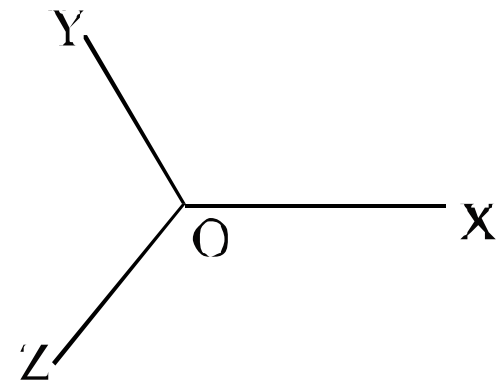

Figure 7. Three dimensional coordinate.

\subsection{Non-Euclidean Geometry}

It is also known as curved geometry. Non- Euclidean geometry is of two types: one is two dimensional and other is three dimensional. Gauss, Bolyai and Lobatchewsky developed two-dimensional curved geometry of surface independently. Riemann developed three dimensional coordinate and also N-dimensional coordinates. Riemannian metric is known as line element and his $\mathrm{N}$-dimension may be called round coordinates instead of spherical coordinate, otherwise there may be confusion between the two. But its a matter of regret that the prospect of Riemann was not understood by mathematicians of his age and even by present age. Contemporary mathematician comments about Riemann that 'Non-Euclidean geometry by Riemannian had been considered utterly brilliant but utterly impractical' (Strathern, 1997) and criticism was very harsh from relativity 'if we would choose a special non-Euclidean geometry of this kind to represent the physical world we should simply be constituting one evil for another' (Born, 1962). This was injustice to Riemann. N-dimensional geometry has possibility. When fixed numbers are added with each Cartesian coordinates $(\mathrm{x}, \mathrm{y}, \mathrm{z})$, it becomes $\mathrm{N}$-dimension coordinates which is round coordinate. In present perspective of the study when time is added with round coordinates, it gets spherical motion. This spherical motion stands to single dimension. This metric is known as line element that is used in spherical geometry by which we can proceed to solve the riddle of unification theory and unified force. Hawking says, 'Thanks to Riemann's investigation of $\mathrm{N}$-dimensional metrical spaces' (Hawking, 2007). Riemann drew line element from Pythagorean law of right angled triangle where

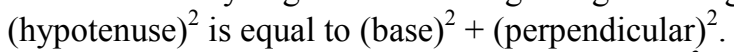

Example of three dimensional line element, $d s^{2}=d x^{2}+d y^{2}$ $+d z^{2}$.

Example of N-dimensional line element: $\mathrm{ds}^{2}=\mathrm{g}_{\mathrm{ijk}} \mathrm{dx} \mathrm{x}^{\mathrm{i}} \mathrm{dx} \mathrm{x}^{\mathrm{j}}$ $\mathrm{dx}^{\mathrm{k}}(\mathrm{i}, \mathrm{j}, \mathrm{k}=1,2,3)$.

$\mathrm{N}$-dimensional coordinates is shown in figure-8.

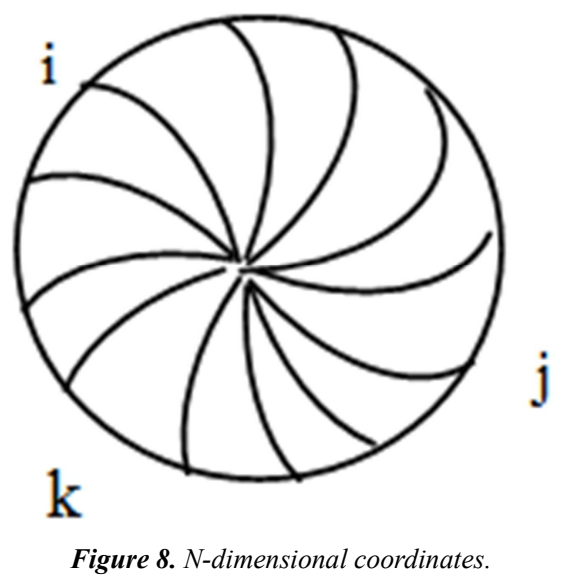

\subsection{Four-Dimensional Geometry}

Minkowski and Einstein added time with three dimensions of space and developed four-dimensional geometry. Minkowski says, 'space by itself and time by itself are doomed to fade away into mere shadows, and only a kind of union of the two preserve an independent reality'-(Good, 1974). Einstein says, 'The world is composed of individual events, each of which is described by four numbers, namely, three space coordinates $\mathrm{x}, \mathrm{y}, \mathrm{z}$ and a time coordinates, the time value is ' $t$ ' (Einstein, 1961).

Einstein worked with four dimensions which have three dimensions of space and one of time using Lorentz transformation. Einstein used four dimensions in his special relativity. But it was not in the form of spherical motion. Einstein presents this law differently in special relativity as $\mathrm{m}=\mathrm{m} 0 / \sqrt{1-v 2 / c 2}$ which tells us how the relativistic mass $\mathrm{m}$ of a body moves at a speed $\mathrm{v}$. We see at once that when $\mathrm{v}$ $=0$, the body then being at rest, we obtain $\mathrm{m}=\mathrm{m} 0$, the rest mass. More generally, as $\mathrm{v} / \mathrm{c} \rightarrow 0$, we find $\mathrm{m} \rightarrow \mathrm{m} 0$, which is the Newtonian limit of the more general expression for the relativistic mass $\mathrm{m}$ ( Resnick, 1968). Here we see a compromise with Newtonian limit instead of spherical motion.

\subsection{Spherical Geometry}

Concept of spherical motion is somewhat difficult. Mankind requires three thousand of years to develop the spherical geometry. Mathematicians developed spherical motion from linear motion, though the concept of linear motion is not consonance with the spherical motion of the universe. 'Space-time is curved, warped, rather than flat, their paths appear to be bent (Hawking, 1996). 'Gravitation has spherical motion otherwise earth would not move around the sun (Tajuddin and Biswas, 2015). 'By gravitation static body stands to mass and moving body stands to acceleration( Tajuddin and others, 2008).Gravitation gives inertia mass to the body and as a result body gets round shape due to the spell of gravitation. Again gravitation helps the body to move spherical way.

As we already have three categories of coordinates, may be transformed to spherical motion adding time with them. First, Riemann's transformation of three dimensional 
Cartesian coordinates (x, y, z) into N-dimensional coordinates, second, transformation of Cartesian coordinates $(\mathrm{x}, \mathrm{y}, \mathrm{z})$ into polar coordinates $(r, \theta, \phi)$, and third, Einstein's metric tensor. These three coordinates are transformed from linear to spherical coordinates and hence they have limitations for many reasons and may not be applied in the field properly.

Examples of the coordinates:

1) N-dimensional spherical tensor: $\mathrm{ds}^{2}=g_{i j \mathrm{k}} d x^{\mathrm{i}} d x^{\mathrm{j}} d \mathrm{x}^{\mathrm{k}}$ $\mathrm{dt}^{2}(\mathrm{i}, \mathrm{j}, \mathrm{k}=1,2,3,4)$

2) Three dimensional Cartesian coordinates $(\mathrm{x}, \mathrm{y}, \mathrm{z})$ into $(r, \theta, \phi)$ as Polar spherical tensor:Example: $d s^{2}=d r^{2}+r^{2} d \theta^{2}+r^{2} \sin ^{2} \theta d \phi^{2}$

3) Einstein worked with the traditional system of geometry that had last development with the method of Bianchi Identities. This tensor turns to zero tensor of four dimensions joining one of time with three of space. Thus Einstein commits the same fallacy what his predecessors faced. Perhaps Einstein did it without his knowing mind because he was not aware of spherical motion of four dimensions, and of time if it is added with space, actually it would revolve. His tensor was also defective as he used zero tensor in his metric. Actually time remains separated in his practice. Coordinates of four dimensional coordinates is shown in figure -9 .
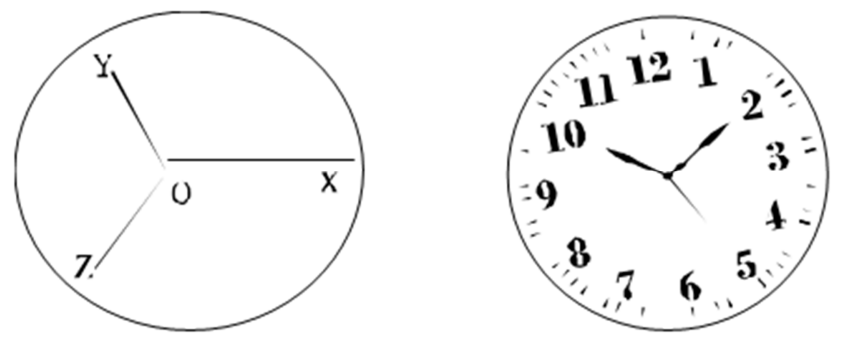

Figure. 9. Four dimensional coordinate by Einstein.

Example: Einstein's four dimensional metric: $\mathrm{ds}^{2}=-\mathrm{dx}^{2}-$ $d y^{2}-d z^{2}+c^{2} d t^{2}$

\subsection{Spherical Geometry of Unified Force}

Non-Euclidean two dimensional spherical geometry was developed by Gauss and others and subsequently three dimensional geometry by Riemann in coordinate system. Schwarzschild developed external solution using polar coordinate. But spherical geometry of unified force is developed by the first author depending on circle as unified force with four properties of matter such as mass of the body, radius of circle, cyclic frequency and time. Here coordinate system is abandoned. Thus spherical geometry of single dimension is developed as line element for unified force. It has validity, objectivity and usability. It is a single law that may be used for law of applied force or law of gravitation. It has supremacy over three previous mentioned spherical coordinates (vide: AJPA, 2015; 3(3)69-76). Line element of unified law is shown in table-1.
Table 1. Line element of unified law.

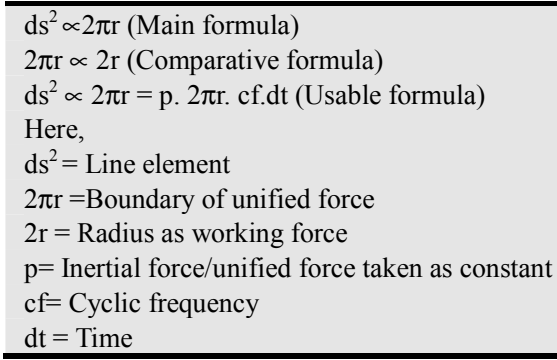

If a motion curves every moment, it stands to spherical motion (vide: how motion stands to spherical). How a round body moves easily in spherical way is shown in figure- 10 .

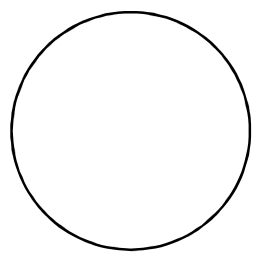

Figure. 10. Spherical motion of round body.

\section{Structure of Unified Force}

At first, four basic forces of quantum mechanics form unified force which may be called four in one or force of single dimension and simultaneously four properties of matter such as radius of circle, mass of the body, cyclic frequency and time join the unified force and make it workable. Thus five forces make unified force (vide: chapter2). Four properties of matter are discussed in chapter-19.

\subsection{Unified Law}

Unified force is used in two ways in two names such as in the form of gravitational attraction in high space and in the form of inertial force in the surface of the earth and air. The planets are attracted by unified force and vehicles are drawn by inertial force. Unified force itself is a spherical force. Unified force of five forces makes unified law. It has somewhat similarity with exterior solution of Schwarzschild solution. Comparison of exterior solution with unified law is shown as $\left(\mathrm{dr}^{2}+\mathrm{r}^{2} \mathrm{~d} \theta^{2}+\mathrm{r}^{2} \sin ^{2} \theta \mathrm{d} \phi^{2}\right)=2 \pi \mathrm{r}$ means boundary of the unified force, $r$ means radius that controls the boundary and $\mathrm{t}=$ time).

Example and explanation unified law is shown in para16.5:

\subsection{Meaning of Unified Force}

The idea of line element is taken from Radian of Trigonometry where circumference $=2 \pi \mathrm{r}$ (circumference /diameter $=\pi$, or, circumference $=\pi d=2 \pi \mathrm{r}$ ). Here the concept $2 \pi \mathrm{r}$ represents the boundary of the unified force whose radius ' $r$ ' controls the boundary. In the formula, $2 \pi r$ means unified force and ' $r$ ' means radius that controls unified force spherically. Here four properties of matter such as radius of the circle, mass of the body, cyclic frequency 
and time join unified force and as a result reaction becomes greater than action. Example: how unified force work united is shown in figure 11.

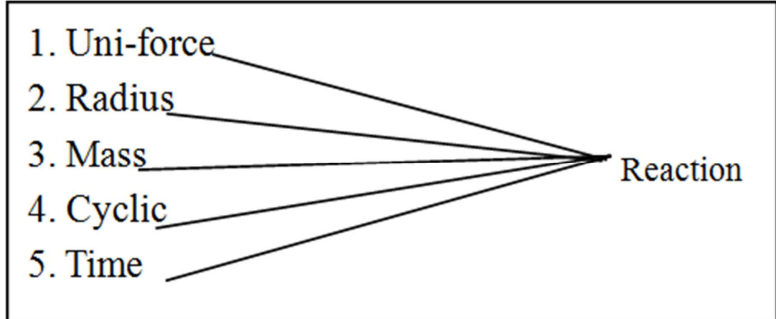

Figure 11. Reaction of unified force is greater than action.

\subsection{Comparison of Unified Law with Classical Law}

In comparative equation, motion in gravitational field is compared with that of non-gravitational field. Here a motion in gravitational field moves $2 \pi \mathrm{r}$ (circumference) in a circle within a fixed time, that motion in non-gravitational field moves equivalent to $2 \mathrm{r}$ (diameter) of that circle. The motion is linear and uniform in non-gravitational field but that of in gravitational field is spherical and accelerated. Newtonian's laws of motion are linear and uniform but unified motion is spherical and accelerated. This comparison is shown in Figure 12.

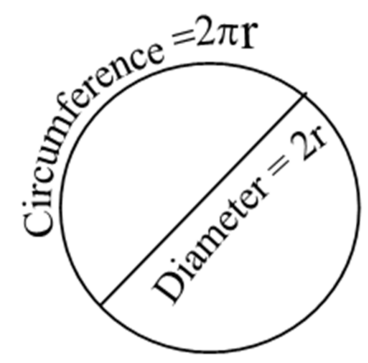

Figure 12. Comparison between linear and spherical motion in a circle.

\subsection{Explanation of Unified Forces}

At first four basic forces form unified force through interaction and at the same time four properties of matter such as radius of the circle, mass of the body, cyclic force and time join unified force. Here reaction is greater than action because unified force works in cyclic way. Suppose a vehicle on earth and a planet in high space use unified force respectively.

\subsection{Principle of Unified Force}

Unification theory has its own philosophy. In gravitational field all bodies remain in the form of balance or round shape such as stars, planets, globe etc. As a result body moves by less force rather than classical force. This is the way buses, trains and vehicles move by less force on wheels. Whatever force is applied here, it would move the body once and extra force would supply by cyclic frequency. Thus the applied force would be considered as constant. The logic is that inertia mass derives from gravitational mass if gravitational force is constant, inertia force would be constant. Schwarzschild in his exterior solution exhibits mass and gravitation both as constant. If more force is applied more advantages will not be added with reaction because the cyclic frequency is there for supplying additional force. Gravitation influences inertia mass on the earth in two ways that is, gravitation static body stands to mass and moving body stands to acceleration (Tajuddin et al., 2007 and 2008)..

In classical physics of non-gravitational field, one body acts upon other in the form of proportion of their masses. Suppose, a body of 40 grams, acts upon another body of 10 grams, the reaction would be $4 \mathrm{~cm} /$ second $^{2}$.

\section{Four Properties of Matter}

At first unified force is create by four basic forces(vide: chapter-2) and again four properties of matter such as mass of the body, radius of circle, cyclic frequency and time join the unified force and make it workable (chapter-18). Here four properties of matter are discussed

\subsection{Mass of the Body}

Mass of the body is a factor. In physics it is known as applied force. Massive body requires more force. The planets move around the sun in sequence of distance due to their density of mass. It goes with the Law of inertia by Newton.

\subsection{Radius of Circle}

A straight line from centre to any point on circumference in a circle is known as radius or Schwarzschild radius. Schwarzschild introduces the idea of radius in his solution. Radius controls unified force. Importance of radius is shown in the formula $\left(d s^{2} \propto 2 \pi r\right)$ of unified force.

\subsection{Cyclic Frequency}

Unified force moves the body spherical way. A vehicle rotates on wheel several times before resuming full motion which is known as cyclic motion or spherical motion. After cyclic motion body gets uniform motion. Suppose, a vehicle moving 8 times gets full motion. This 8 time movements are known as cyclic motion. Mathematical analysis would be $2 \pi r \times 8=16 \pi r$.

\subsection{Time}

Motion is always counted in respect of time. Time is always involved with motion. Time is counted as how many times. Here $t$ is used as time (Tajuddin, 2014)

\section{Conclusion}

Bondage of long range particles of spin $1 / 2$, and spin $1 / 3$ and short range particles of spin 0 , spin 1 and spin 2 form unified force, of them first category belongs to gravitation group particles and second category belongs to short range basic forces lying in the inertia of mass of the earth and it is confirmed by previous researches done by quantum 
mechanics, theoretical physics, different observations and mathematical analysis (vide: section- 3, 4). If the bondage of two categories, that is, the four basic forces is right, unified force is right and spherical motion of the universe is right. Unified force has own existence and own functioning capacity other than four basic forces (vide: sections 1-4). Unified force is an ultimate scale to discover new truth and avoidance of error of existing laws of physical sciences. Now it is our foremost duty to discover new scientific truths and discard the false law from our physical sciences in the light of unified force. Unified theory can do immense benefit of mankind

\section{References}

[1] Abbot, A,F. 'Physics',(1997), fifth edition, Heinemann Education, Oxford, London.

[2] Born, M. (1962). "Einstein's Theory of Relativity", Dover Publications Inc. New York.

[3] Einstein, A. (1961), 'Relativity: The Special and the General Theory,' Three Rivers Press, New York.

[4] Good, R.H. (1974). "Basic Concepts of Relativity", East-West Edition, New Delhi, India.

[5] Hawking, S.W. (1996). "A Brief History of Time", Bantam Books, New York.

[6] Hawking, S.W. (1993), 'Black Holes and Baby Universe and Other Essays', Bantam Books, New York, London.
[7] Hawking, S. W. (2007). "The Essential Einstein: His Greatest Works", Penguin Books, London, U.K.

[8] Resnick. R. (1968), 'Introduction to Special Relativity', John Wiley and Sons, Inc. New York.

[9] Resnick, R. and Hallidy, D.(1966), "Physics, Part-1", Wiley Eastern Limited, New Delhi

[10] Strathern, P. (1997). "Einstein and Relativity", Arrow Books, UK.

[11] Synge, J. L. and Schild, A. (1978). "Tensor Calculus", Dover Publications Inc. New York. P. 96

[12] Tajuddin,M and Biswas, M. H.A. (2015).Einstein: "Unification Theory and Unification of Basic Forces", American Journal of Physics and Application, New York, 2015;3(3)69-76.

[13] Tajuddin, M. (2014). "Einstein: Unification of Basic Forces" (in Bengali), Utso Publication, Dhaka, Bangladesh.

[14] Tajuddin, M. Biswas, M. H. A. and Uddin, M. S. (2007). 'New Aspects of Principle of Equivalence', Acta Ciencia Indica, Vol. XXXIV M, No. 4, pp. 1931-1939.

[15] Tajuddin, M Biswas, M. H. A. and Islam, A. (2008), 'Review of the Theory of Relativity.' Journal of Applied Sciences Research, 4(1): 32-39.

[16] Tajuddin, M. and Biswas, M. H. A. (2005). 'Unification of the Theory of Albert Einstein by Cyclic Theory', Asian J. Inform. Tech., 4(10): 962-970.

[17] Vasudeva, D.N. Fundamentals of Magnetism and Electricity, S.Chand \& Company Ltd. Ramnagar, New Delhi, 1983. 